\title{
The Effect of Obesity on Outcomes of Ventral Hernia Repair: A Propensity Score Matching Analysis
}

\section{Neil P. Borad and Aziz M. Merchant}

Department of Surgery, New Jersey Medical School, Rutgers University, Newark, NJ, USA

'Correspondence to:

Aziz M. Merchant, MD, FACS

185 South Orange Avenue

Suite MSB G530, Newark, NJ 07103, USA

Tel: $973-972-0072$

Fax: 973-972-6083

E-mail: Aziz.merchant@rutgers.edu

Received: January 08, 2017

Accepted: March 10, 2017

Published: March 13, 2017

Citation: Borad NP, Merchant AM. 2017. The Effect of Obesity on Outcomes of Ventral Hernia Repair: A Propensity Score Matching Analysis. J Obes Chronic Dis 1(1): 6-14.

Copyright: (C) 2017 Borad and Merchant. This is an Open Access article distributed under the terms of the Creative Commons Attribution 4.0 International License (CC-BY) (http:// creativecommons.org/licenses/by/4.0/) which permits commercial use, including reproduction, adaptation, and distribution of the article provided the original author and source are credited.

Published by United Scientific Group

\begin{abstract}
Purpose: To determine whether overweight body mass index (BMI) impacts adverse 30-day outcomes in ventral hernia repair (VHR).

Study design: Observational retrospective cohort study of a prospectively collected database.

Methods: Data from the National Surgical Quality Improvement Program were extracted for patients undergoing VHR from 2005-2014. Patients were stratified into five BMI $\left(\mathrm{kg} / \mathrm{m}^{2}\right)$ categories [normal (18.5-24.9), pre-obese (25.029.9), obese I (30.0-34.9), obese II (35.0-39.9), and obese III ( $\geq 40)]$ and were compared for significant differences in pre-operative characteristics. Propensityscore matching evenly distributed confounding covariates amongst BMI categories. Multivariate logistic regression was used before and after matching to isolate the impact of BMI on the primary outcome of any 30-day morbidity and secondary outcomes of 30-day wound-, respiratory complications, and mortality.

Results: $84.1 \%$ of VHR patients in the cohort $(168,214)$ were overweight $(\mathrm{BMI} \geq 25)$. In the unmatched analyses, only obese I-III patients showed increased odds of any 30-day morbidity while every overweight BMI category was associated positively with 30 -day wound complications. After propensityscore matching, every overweight category showed increased odds for both any 30 -day complication $\left[\mathrm{OR}_{\text {Preobese }} 1.12, p=0.008 ; \mathrm{OR}_{\text {obeselII }} 1.64, p<0.0001\right]$ as well as for wound-based complication in a dose-dependent manner.
\end{abstract}

Conclusions and clinical relevance: Increased BMI is a modifiable risk factor for adverse surgical outcomes in patients undergoing VHR. With obesity rates on the rise, these findings can be used by surgeons to assist in the selection and evaluation of obese patients being considered for VHR and more importantly, be used to educate patients presenting with VHR about the risks of elective repair.

\section{Keywords}

Obesity, Body mass index, Ventral hernia repair, 30-day outcomes, Propensity score matching, NSQIP

\section{Introduction}

With over 350,000 procedures annually, ventral hernia repair (VHR) remains one of the most common surgeries in the United States [1]. Given that over a third of the nation's population is obese [2], a significant fraction of patients who approach general surgeons for ventral hernia management present with obesity and obesity-related comorbidities. Obesity has been studied to be significantly associated with the initial development of both primary and incisional ventral hernias [3-5]. Moreover, hernia recurrences, the Achilles heel of hernia repair, 
range from 24-43\% [4] and can be attributed to a number of risk factors, including obesity [6]. This finding, in combination with the fact that preventing even $1 \%$ of hernia recurrences amounts to over $\$ 30$ million in healthcare savings $[7,8]$ emphasizes the importance of investigating obesity's impact on all aspects of hernia repair.

A number of recent studies have found an association between obesity and post-operative complications after ventral hernia repair [9-12]. While some of these studies looked at outcomes such as length of stay and recurrence, others investigated metrics related to quality of care such as postoperative wound complications. However, specific limitations such as using patients from a single institution $(n<150)$ and excluding obese patients with BMI's between 25-35 [11, 12] contribute to some uncertainty in these studies findings. Furthermore, even though the remaining studies used larger databases, they either included inguinal hernias in addition to ventral hernias [10] or only looked VHR procedures from 2009-2012 in the context of open versus laparoscopic repair. While increasing body mass index was associated with a greater likelihood of post-operative complications, these studies used multivariate regression without an adjustment for pre-operative factors that contributed to selection bias in the results. This study aims to improve upon these shortcomings by using the American College of Surgeons National Surgical Quality Improvement Program (ACS-NSQIP) from 20052014 to evaluate the isolated impact of body mass index on adverse 30-day surgical outcomes such as overall morbidity, wound morbidity, respiratory morbidity, and mortality in ventral hernia repair patients using propensity-score matching.

\section{Materials and Methods}

\section{Data source and study population}

Institutional Review Board approval was obtained. This study used the ACS-NSQIP database. The ACS-NSQIP database contains de-identified and robust data consisting of demographic variables, preoperative risk factors, intraoperative information, and 30-day postoperative outcomes. Given the validated and prospective nature of the program, the ACSNSQIP database serves as a comprehensive source of national data for clinical studies investigating surgical outcomes [13].

For the purposes of this study, we first identified every patient who underwent ventral hernia repair by the principal procedure CPT codes of 49560 (initial incisional/ventral hernia), 49565 (recurrent incisional/ventral hernia), 49570 (epigastric hernia), 49585 (umbilical hernia), 49590 (spigelian hernia), 49652 (laparoscopic repair of ventral, umbilical, spigelian, or epigastric hernia), 49564 (laparoscopic repair of Initial incisional/ventral hernia), 49656 (laparoscopic repair of recurrent incisional/ventral hernia). These CPT codes were sampled from the ACS-NSQIP database every year from 2005-2014 to extract patients who underwent ventral hernia repair. At this point, exclusions were made for (1) any patients over 18 years of age, (2) with a body mass index (BMI) greater than 60 or under 18.5 , (3) or missing data for age, BMI, smoking status, or missing 30-day mortality information. The $\mathrm{BMI}>60$ exclusion was made due to a low sample size while the $\mathrm{BMI}<18.5$ exclusion was made due to the study's focus on overweight and obese (BMI > 25) patients only. This final sample served as the baseline cohort from which further stratifications were made prior to statistical analysis (Figure 1).

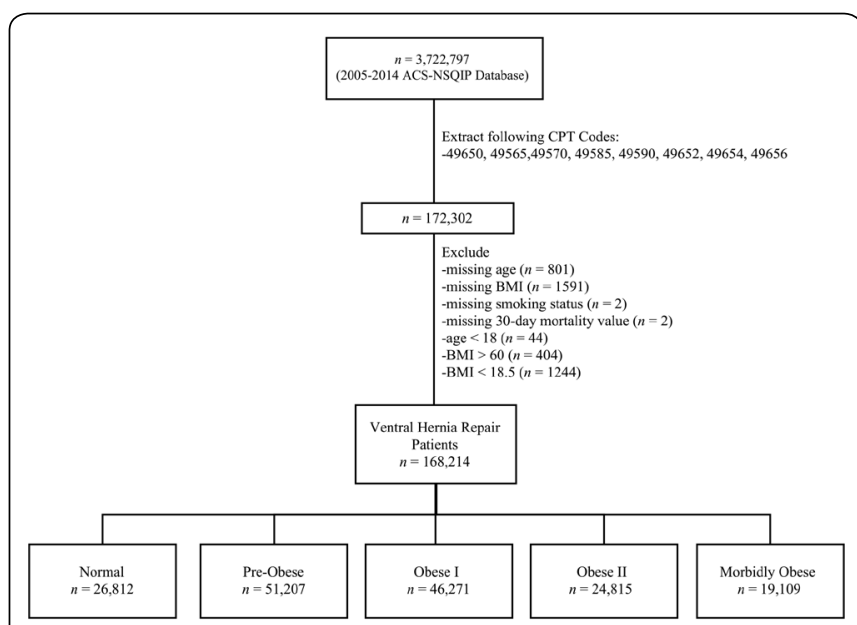

Figure 1: Flowchart of patient selection from ACS NSQIP databases (2005-2014).

\section{BMI categories and other predictor variables}

The primary predictor variable in this study was body mass index (BMI). BMI was calculated for every patient in our cohort using the height and weight information reported by the NSQIP database. The patients were then stratified into 5 BMI categories based on the World Health Organization's classification system (normal: 18.5-24.9 kg/m², pre-obese: 25.0-29.9 kg/m², obese I: $30.0-34.9 \mathrm{~kg} / \mathrm{m}^{2}$, obese II: $35.0-$ $39.9 \mathrm{~kg} / \mathrm{m}^{2}$, and obese III: $\geq 40 \mathrm{~kg} / \mathrm{m}^{2}$ ).

Data was also collected for demographic covariates including age, sex, and smoking status. Data for preoperative data was collected for the following variables: surgery type (laparoscopic or open), emergency operation, preoperative cardiac risk (presence of hypertensive medications, congestive heart failure, myocardial infarction, percutaneous coronary intervention or angioplasty, angina, or cardiac surgery), diabetes, ventilator dependence, history of chronic obstructive pulmonary disease (COPD), chronic steroid use, dialysis, and American Society of Anesthesiology (ASA) Class.

\section{Outcomes of Interest}

The primary outcome measured was 30-day overall morbidity. 30-day overall morbidity reflects the presence of any major complication within 30-days of VHR and is a summation of wound, respiratory, cardiac, central nervous system, genitourinary, and other occurrences (Table 1). Secondary outcomes measured were 30 -day wound morbidity, 30-day respiratory morbidity, and 30-day mortality and were selected due to their frequency (Table 2). Patients with at least one morbidity complication within 30-days were considered to have a positive 30 -day overall morbidity. Independent subgroup analyses of 30-day wound morbidity, 30-day respiratory morbidity, and 30-day mortality were performed because of significant differences in their incidence between normal BMI patients and overweight/obese BMI patients (Table 2). Wound morbidity was determined based 
on patients having at least one of the following occurrences within 30-days: superficial incisional surgical site infection (SSI), deep incisional SSI, organ/space SSI, wound disruption, or peripheral nerve injury. Respiratory morbidity was determined based on subjects having at least one of the following occurrences within 30-days: pneumonia, unplanned intubation, pulmonary embolism, and ventilator dependence greater than 48 hours.

Table 1: Definition of predictor variables and postoperative occurrences.

\begin{tabular}{|l|l|}
\hline Occurrence Type & Definition \\
\hline Definitions of postoperative occurrences \\
\hline $\begin{array}{l}\text { 30-day Overall } \\
\text { morbidity }\end{array}$ & $\begin{array}{l}\text { Summation of wound, respiratory, genitourinary, } \\
\text { central nervous system, cardiac, and other occurrences. } \\
\text { Patients with at least one morbidity occurrence } \\
\text { considered to have a 30-day overall morbidity }\end{array}$ \\
\hline Wound morbidity & $\begin{array}{l}\text { At least one of the following occurrences: } \\
\text { Superficial SSI } \text {, deep incisional SSI, organ/space } \\
\text { SSI, wound disruption, peripheral nerve injury }\end{array}$ \\
\hline $\begin{array}{l}\text { Respiratory } \\
\text { morbidity }\end{array}$ & $\begin{array}{l}\text { At least one of the following occurrences: } \\
\text { Pneumonia, unplanned intubation, pulmonary } \\
\text { embolism, ventilator dependence > than 48 h }\end{array}$ \\
\hline Cardiac morbidity & $\begin{array}{l}\text { At least one of the following occurrences: Cardiac } \\
\text { arrest requiring CPR, 30-day occurrence of } \\
\text { myocardial infarction }\end{array}$ \\
\hline infection & $\begin{array}{l}\text { At least one of the following occurrences: Stroke/ } \\
\text { CVA or Coma greater than 24 Hours }\end{array}$ \\
\hline CNS morbidity
\end{tabular}

\section{Statistical analysis and propensity matching}

Patients in the 4 BMI study groups (pre-obese, obese I-III) were each compared to patients with normal BMI for significant differences in demographic and pre-operative covariate distributions via the student's $t$-test for continuous variables, chi-square test for categorical variables, or the Wilcoxon-Mann-Whitney U-test for variables with nonnormal distributions and unequal variances (Table 3). The frequency of each 30-day clinical outcome variable was also stratified by BMI category and compared with the normal BMI group using Fisher's exact tests for binary outcome variables and the chi-square test as appropriate (Table 2).

Given the retrospective design of our study, there was a high likelihood of selection bias within each of the $5 \mathrm{BMI}$ category samples that needed to be acknowledged. Propensityscore matching is a well-established statistical technique used to reduce the effects of selection bias in an observational study [14]. First, propensity scores were generated for every patient within each of the $5 \mathrm{BMI}$ groups using their individual baseline demographics and preoperative characteristics. A patient's propensity score, in the context of this study, reflected the probability of that patient being classified in a particular BMI group given their individual pre-clinical characteristics. Therefore, patients with similar propensity scores were considered to have a comparable distribution of pre-operative baseline characteristics and were more importantly, considered to be equally likely to be a normal BMI patient or an overweight/ obese patient. Once propensity scores were generated, a greedy 5:1-digit match algorithm was used to group patients with similar propensity scores in either the normal BMI category or in one of the 4 overweight/obese BMI categories. A greedy algorithm matches a set of $\mathrm{X}$ cases to a set of $\mathrm{Y}$ controls in a set of $\mathrm{X}$ decisions [15]. Every overweight patient in the sample (the "case") was ordered by their respective propensity scores and sequentially matched to a normal BMI patient (the "control") with the nearest propensity score to produce two "matched" samples of overweight BMI patients and normal BMI patients. The same technique was used to match patients in the obese I-III categories to normal BMI patients. Student t-tests and chi-square tests were used to confirm the notion that the samples of "matched" patients were expected to have similar distributions of baseline preoperative characteristics

Table 2: 30-day Surgical Outcomes following Ventral hernia repair stratified by BMI category.

\begin{tabular}{|l|c|c|c|c|c|c|c|c|c|}
\hline \multirow{2}{*}{ Variable } & Normal & Pre-Obese & & Obese I & & Obese II & & Obese III \\
\cline { 2 - 23 } & $(N=26812)$ & $(N=51207)$ & $P$-value & $(N=46271)$ & $P$-value & $(N=24815)$ & $P$-value & $(N=19109)$ & $P$-value \\
\hline All Morbidities (30-day) & $4.04 \%$ & $4.33 \%$ & $\mathbf{0 . 0 4 9 3}$ & $5.31 \%$ & $<.0001$ & $6.62 \%$ & $<.0001$ & $9.66 \%$ & $<.0001$ \\
\hline Wound Morbidity (30-day) & $1.81 \%$ & $2.35 \%$ & $<.0001$ & $3.25 \%$ & $<.0001$ & $4.38 \%$ & $<.0001$ & $6.75 \%$ & $<.0001$ \\
\hline Resp. Morbidity (30-day) & $1.11 \%$ & $1.06 \%$ & 0.4798 & $1.11 \%$ & 0.9726 & $1.33 \%$ & $\mathbf{0 . 0 2 6 3}$ & $1.77 \%$ & $<.0001$ \\
\hline Cardiac Morbidity (30-day) & $0.21 \%$ & $0.22 \%$ & 0.6954 & $0.22 \%$ & 0.6576 & $0.21 \%$ & 0.9335 & $0.21 \%$ & 0.9914 \\
\hline CNS Morbidity (30-day) & $0.04 \%$ & $0.05 \%$ & 0.6362 & $0.07 \%$ & 0.1949 & $0.06 \%$ & 0.4359 & $0.06 \%$ & 0.4044 \\
\hline GU Morbidity (30-day) & $0.92 \%$ & $0.86 \%$ & 0.3489 & $0.96 \%$ & 0.6260 & $1.08 \%$ & 0.0700 & $1.50 \%$ & 0.0834 \\
\hline Mortality (30-day) & $0.32 \%$ & $0.20 \%$ & $\mathbf{0 . 0 0 0 6}$ & $0.19 \%$ & $\mathbf{0 . 0 0 0 4}$ & $0.17 \%$ & $\mathbf{0 . 0 0 0 4}$ & $0.25 \%$ & 0.1241 \\
\hline
\end{tabular}


(Table 4). The matched samples should only differ in their BMI classification, which allows for isolation of BMI's impact on the 30-day surgical outcome variables.

Using the unmatched samples first, each outcome variable (30-day morbidity, 30-day wound, 30-day respiratory, and 30day mortality) was first modelled using multivariate logistic regression to assess the effect of a particular BMI category on the likelihood of having the given outcome. For each model, the model chi-square $\left(\mathrm{X}^{2}\right)$, odds ratio (OR) with $95 \%$ confidence interval (CI), and $p$-value were calculated. While the model $\mathrm{X}^{2}$ and $\mathrm{p}$-value indicated statistical significance, the OR quantified the impact of being in a particular BMI category on the likelihood of a patient suffering an outcome.

Multivariate logistic regression was used again on the matched samples to re-assess the effect of a particular BMI category on the likelihood of having a particular 30-day surgical outcome without the influence of selection bias. The $\mathrm{OR}, \mathrm{CI}$, and $p$-values were obtained for each BMI category to assess their predictive value on the odds of suffering a 30-day outcome.

Significance was determined using the a priori alpha level of 0.05. All analyses were done using Statistical Analysis System (SAS) version 9.4 (SAS Institute, North Carolina).

\section{Results}

Demographics, pre-operative characteristics, and surgical outcomes

A total of 168,214 patients undergoing ventral hernia repair from 2005-2014 were identified from the ACS NSQIP database (Figure 1). The most represented CPT codes were 49560 (34.9\%), 49585 (32.2\%), 49565 (10.2\%), and 49652 (9.48\%). Table 3 summarizes all the patient demographic and baseline preoperative characteristics stratified by BMI category. From the overall sample, 26,812 (15.9\%) patients were of normal BMI while 141,402 (84.1\%) were overweight or obese. The most represented BMI categories were the preobese (30.4\%) and obese I (27.5\%) groups. The average study patient was a 53.8-year-old male with a BMI of 31.5 and ASA Class II. Pre-operative cardiac risk (44.6\%) and diabetes (13.7\%) were the major comorbidities in the total sample at the time of ventral hernia repair. $80.9 \%$ of patients were treated using open ventral hernia repair and only $1.83 \%$ were emergent operations.

Table 2 lists the frequency of 30-day surgical outcomes including 30-day mortality or one of the 30-day morbidity sub-classifications found in Table 1. Outcome occurrences were stratified by BMI categories as well. $5.5 \%$ of the total sample suffered an overall morbidity within 30 days of VHR while only $0.22 \%$ of the total sample died within 30 days of VHR. Out of the 9242 morbidities suffered, woundbased (60.27\%) and respiratory-based (21.85\%) were the most common. Patients with normal BMI and pre-obese/ obese I-III patients differed significantly $(p<0.05)$ in All morbidities, wound morbidity, respiratory morbidities, and 30day mortality (Table 2). There was not a significant difference in the frequency of Cardiac, CNS, or GU 30-day morbidities between normal BMI patients and any of the pre-obese/obese I-III categories.

Comparison of baseline demographic and preoperative information distributions

Comparing demographics and preoperative characteristics between pre-obese / obese I-III patients and normal BMI patients in the unmatched sample showed that obese II patients did not differ significantly in ventilator dependence $(p=0.231)$ and emergent operations $(p=0.23)$ while obese III patients did not differ significantly in ventilator dependence $(p=0.498)$ and history of COPD $(p=0.552)$ compared to normal BMI patients. However, statistically significant differences $(p<0.05)$ were found between normal BMI and pre-obese/obese I-III patients in the following variables: age, sex, mean BMI, smoking status, surgery type, preoperative cardiac risk, diabetes status, chronic steroid use, dialysis, and in overall ASA Class distribution (Table 3).

The 5:1-1-digit greedy match with propensity scores produced 4 groups with normal BMI patients matched to one of the four Obesity categories. Each of the 4 groups contained an equal number of normal BMI patients and pre-obese, obese I, obese II, or obese III patients $(n=25,043 ; 45,425 ; 34,340$; 25,728 , respectively). Table 4 shows demographic and preoperative characteristics after propensity-score matching. The four matched cohorts (normal vs pre-obese, normal vs obese I, normal vs obese II, and normal vs obese III) no longer had significant differences in any of the preoperative variables $(p>$ 0.05 for all) and reflected a random distribution of covariates within every BMI category.

\section{Multivariate analysis of morbidity and mortality}

Multivariate generalized logistic regression with the five BMI category variables as well as additional demographic and preoperative covariates was used on the unmatched cohort to assess the impact of a patient's particular BMI category on their likelihood of any 30-day morbidity, 30-day wound morbidity, 30-day respiratory morbidity, and 30-day Mortality after VHR. Patients from only the obese I-III BMI groups were found to have a statistically increased likelihood of any 30 -day morbidity with patients in the pre-obese BMI group having a non-significant odds ratio $(\mathrm{OR}=1.08, p=0.054)$ (Table 5). For the significant BMI categories, the odds ratios increased as the BMI category increased, with patients in the obese I category having a 33\% increased odds of 30-day morbidity while patients in the obese III category having over $150 \%$ increased odds $\left(\mathrm{OR}_{\text {obeseI }} 1.33, p<.0001\right.$ O OR $_{\text {obeseIII }} 2.54$, $p<.0001)$. Every BMI category was a significant positive predictor of suffering a 30-day wound morbidity in an increasing pattern as well. Patients in the pre-obese category had a $30 \%$ increased likelihood of a wound complication while patients in the obese III group saw close to a $3 \mathrm{x}$ increase in the odds of a 30-day wound morbidity $\left(\mathrm{OR}_{\text {Preobese }} 1.30, p<.0001\right.$; $\left.\mathrm{OR}_{\mathrm{OIII}} 3.92, p<.0001\right)$. Only patients in the obese III category had a statistically significant increased likelihood of suffering a 30 -day respiratory morbidity $\left(\mathrm{OR}_{\text {obeseIII }} 1.61, p<.0001\right)$. Lastly, patients in the pre-obese and obese I-II groups were found to 
Table 3: Patient demographics and clinical characteristics stratified by Body Mass Index (unmatched cohorts).

\begin{tabular}{|c|c|c|c|c|c|c|c|c|c|}
\hline \multirow{2}{*}{ Variable } & Normal & Pre-Obese & & Obese I & & Obese II & & Obese III & \\
\hline & $(N=26812)$ & $(N=51207)$ & P-value & $(N=46271)$ & P-value & $(N=24815)$ & P-value & $(N=19109)$ & $P$-value \\
\hline Mean BMI $\left(\mathrm{kg} / \mathrm{m}^{\wedge}-2\right)$ & $22.7 \pm 1.6$ & $27.6 \pm 1.4$ & $<.0001$ & $32.3 \pm 1.4$ & $<.0001$ & $37.2 \pm 1.4$ & $<.0001$ & $45.1 \pm 4.5$ & $<.0001$ \\
\hline Mean Patient Age & $54.8 \pm 17.3$ & $55.7 \pm 15.0$ & $<.0001$ & $54.8 \pm 13.4$ & $<.0001$ & $53.4 \pm 12.8$ & $<.0001$ & $50.6 \pm 12.4$ & $<.0001$ \\
\hline Female & $62.5 \%$ & $39.2 \%$ & $<.0001$ & $40.3 \%$ & $<.0001$ & $50.3 \%$ & $<.0001$ & $64.7 \%$ & $<.0001$ \\
\hline Current Smoker (w/in 1 yr) & $22.6 \%$ & $18.7 \%$ & $<.0001$ & $18.5 \%$ & $<.0001$ & $19.2 \%$ & $<.0001$ & $18.9 \%$ & $<.0001$ \\
\hline \multicolumn{10}{|l|}{ Surgery Type } \\
\hline Laparoscopic Hernia & $14.1 \%$ & $16.9 \%$ & \multirow{2}{*}{$<.0001$} & $19.9 \%$ & \multirow{2}{*}{$<.0001$} & $22.7 \%$ & \multirow{2}{*}{$<.0001$} & $24.1 \%$ & \multirow{2}{*}{$<.0001$} \\
\hline Open Hernia & $85.9 \%$ & $83.1 \%$ & & $80.1 \%$ & & $77.3 \%$ & & $75.9 \%$ & \\
\hline \multicolumn{10}{|l|}{ Operative Details } \\
\hline Emergent Operation & $2.0 \%$ & $1.6 \%$ & 0.002 & $1.6 \%$ & 0.001 & $1.8 \%$ & 0.230 & $2.7 \%$ & $<.0001$ \\
\hline \multicolumn{10}{|l|}{ Preoperative Conditions } \\
\hline Pre-Operative Cardiac Risk & $28.5 \%$ & $41.4 \%$ & $<.0001$ & $48.4 \%$ & $<.0001$ & $53.1 \%$ & $<.0001$ & $55.7 \%$ & $<.0001$ \\
\hline Diabetes & $5.6 \%$ & $9.7 \%$ & $<.0001$ & $14.8 \%$ & $<.0001$ & $21.0 \%$ & $<.0001$ & $24.3 \%$ & $<.0001$ \\
\hline Ventilator Dependent & $0.1 \%$ & $0.0 \%$ & 0.026 & $0.0 \%$ & 0.018 & $0.1 \%$ & 0.231 & $0.1 \%$ & 0.489 \\
\hline History of severe COPD & $4.3 \%$ & $3.8 \%$ & 0.001 & $3.8 \%$ & 0.001 & $3.8 \%$ & 0.003 & $4.4 \%$ & 0.552 \\
\hline Chronic steroid use & $4.0 \%$ & $3.4 \%$ & $<.0001$ & $2.9 \%$ & $<.0001$ & $2.4 \%$ & $<.0001$ & $2.2 \%$ & $<.0001$ \\
\hline Dialysis & $1.7 \%$ & $1.1 \%$ & $<.0001$ & $0.8 \%$ & $<.0001$ & $0.8 \%$ & $<.0001$ & $0.7 \%$ & $<.0001$ \\
\hline \multicolumn{10}{|l|}{ ASA Classification } \\
\hline I-No Disturbance & $22.7 \%$ & $13.9 \%$ & \multirow{4}{*}{$<.0001$} & $7.8 \%$ & \multirow{4}{*}{$<.0001$} & $3.6 \%$ & \multirow{4}{*}{$<.0001$} & $1.4 \%$ & \multirow{4}{*}{$<.0001$} \\
\hline II-Mild Disturbance & $49.6 \%$ & $56.2 \%$ & & $58.7 \%$ & & $52.6 \%$ & & $35.4 \%$ & \\
\hline III-Severe Disturbance & $24.7 \%$ & $27.7 \%$ & & $31.5 \%$ & & $41.8 \%$ & & $59.7 \%$ & \\
\hline IV-Life Threatening & $2.7 \%$ & $2.0 \%$ & & $1.7 \%$ & & $1.7 \%$ & & $3.3 \%$ & \\
\hline
\end{tabular}

have a significantly reduced likelihood of suffering a 30-day mortality compared to normal BMI patients $\left(\mathrm{OR}_{\text {Preobese }} 0.61\right.$, $\left.p=0.0006 ; \mathrm{OR}_{\mathrm{OI}} 0.59, p=0.0005 ; \mathrm{OR}_{\text {obeseII }} 0.52, p=0.0005\right)$.

In the matched samples, multivariate generalized logistic regression now showed that pre-obese patients were also significant in predicting any 30-day morbidity, along with obese I-III patients (Table 6). These odds ratios increased as the BMI category increased, with pre-obese patients having a $12 \%$ increase in 30 -day morbidity likelihood and obese III patients having a $64 \%$ increase $\left(\mathrm{OR}_{\text {Preobese }} 1.12, p 0.008\right.$; $\mathrm{OR}_{\text {OII }}$ $1.64, p<.0001)$. Similar findings were seen with 30 -day wound morbidity as well, as every obesity category was statistically significant in increasing the likelihood of suffering a wound complication after VHR. After matching, the patients in the Obesity III no longer had a significantly higher likelihood of suffering a 30-day respiratory morbidity compared to normal $\mathrm{BMI}$ patients $\left(\mathrm{OR}_{\mathrm{OIII}} 0.98, p=0.76\right)$. Finally, patients in every obesity category were still found to have reduced odds of dying after 30-day mortality, with patients in the obese II and III categories having stronger statistical significance (Table 6).

\section{Discussion}

Over $80 \%$ of VHR from 2005-2014 in the NSQIP database were conducted on patients with pre-obese or obese Body Mass Indices. Out of the 9242 morbidities suffered 30day after VHR, the majority were wound based (60.27\%). When stratified by BMI category, the frequency of wound complications increased with increasing BMI category. We found the obese III group $\left(\geq 40 \mathrm{~kg} / \mathrm{m}^{2}\right.$ ) had a significantly increased difference in wound complications than patients with normal BMI ( $\geq 18.5$ to $\leq 25 \mathrm{~kg} / \mathrm{m}^{2}$ ) (Table 2). These findings are consistent with previous literature that found an increased frequency of surgical, wound-related, complications for patients with BMI 40 or over that was significantly different than the frequency of wound-complications in normal BMI patients $[8,12]$. The scientific rationale for higher frequencies of wound-related morbidities such as surgical site infection (SSI) is thought to be from a complex multitude of factors, including tissue hypo perfusion predisposing the obese to SSI via slower collagen synthesis and technical factors such as longer operation times with morbidly obese patients $[16,17]$.

Stratifying patients by BMI category to compare baseline demographic and pre-operative comorbidities between pre-/ obese patients to normal BMI patients revealed significant differences between these groups. pre-obese and obese I-III patients were found to differ significantly in their mean age, sex, smoking status, surgery type, preoperative cardiac risk, diabetes status, chronic steroid use, dialysis, and in overall ASA distribution compared to normal BMI patients. Because some of these comorbidities (i.e. preoperative cardiac risk and diabetes) are associated with not only the primary independent variable (body mass index), but also our outcome variables (30-day overall/wound/respiratory morbidity), any relationship between body mass index and post-operative outcomes has significant confounding that needs to be 
Table 4: Patient demographics and clinical characteristics stratified by Body Mass Index (matched cohorts).

\begin{tabular}{|c|c|c|c|c|c|c|c|c|c|c|c|c|}
\hline \multirow[b]{2}{*}{ Variable } & Normal & $\begin{array}{l}\text { Pre- } \\
\text { obese }\end{array}$ & \multirow[b]{2}{*}{ P-value } & Normal & Obese I & \multirow[b]{2}{*}{$P$-value } & Normal & Obese II & \multirow[b]{2}{*}{$P$-value } & Normal & $\begin{array}{l}\text { Obese } \\
\text { III }\end{array}$ & \multirow[b]{2}{*}{ P-valu } \\
\hline & \multicolumn{2}{|c|}{$(N=25043)$} & & \multicolumn{2}{|c|}{$(N=45524)$} & & \multicolumn{2}{|c|}{$(N=34240)$} & & \multicolumn{2}{|c|}{$(N=25728)$} & \\
\hline Mean BMI $\left(\mathrm{kg} / \mathrm{m}^{\wedge}-2\right)$ & $23 \pm 1.6$ & $28 \pm 1.4$ & $<.0001$ & $23 \pm 1.6$ & $32 \pm 1.4$ & $<.0001$ & $23 \pm 1.6$ & $37 \pm 1.3$ & $<.0001$ & $23 \pm 1.6$ & $45 \pm 4.4$ & $<.0001$ \\
\hline Mean Patient Age & $52 \pm 17.3$ & $53 \pm 15.6$ & 0.212 & $54 \pm 17.2$ & $52 \pm 14.0$ & 0.231 & $56 \pm 16.2$ & $53 \pm 12.4$ & 0.321 & $59 \pm 16.4$ & $49 \pm 12.3$ & 0.056 \\
\hline Female & $59.9 \%$ & $60.2 \%$ & 0.835 & $56.2 \%$ & $56.7 \%$ & 0.433 & $53.0 \%$ & $53.6 \%$ & 0.145 & $58.4 \%$ & $58.8 \%$ & 0.392 \\
\hline Current Smoker (1 yr.) & $23.5 \%$ & $23.6 \%$ & 0.689 & $23.7 \%$ & $23.6 \%$ & 0.651 & $23.0 \%$ & $23.3 \%$ & 0.573 & $22.0 \%$ & $21.9 \%$ & 0.786 \\
\hline \multicolumn{13}{|l|}{ Surgery Type } \\
\hline Laparoscopic Hernia & $14.5 \%$ & $14.3 \%$ & \multirow{2}{*}{0.495} & $14.7 \%$ & $16.3 \%$ & \multirow{2}{*}{0.542} & $15.7 \%$ & $20.9 \%$ & \multirow{2}{*}{0.801} & $16.2 \%$ & $21.6 \%$ & \multirow{2}{*}{0.921} \\
\hline Open Hernia & $85.5 \%$ & $85.7 \%$ & & $85.3 \%$ & $83.7 \%$ & & $84.3 \%$ & $79.1 \%$ & & $83.8 \%$ & $78.4 \%$ & \\
\hline \multicolumn{13}{|l|}{ Operative Details } \\
\hline Emergent Operation & $2.0 \%$ & $2.0 \%$ & 0.949 & $2.1 \%$ & $2.2 \%$ & 0.243 & $2.3 \%$ & $2.2 \%$ & 0.531 & $2.6 \%$ & $2.5 \%$ & 0.502 \\
\hline \multicolumn{13}{|l|}{ Preoperative Conditions } \\
\hline Pre-Op. Cardiac Risk & $30.4 \%$ & $30.4 \%$ & 0.985 & $33.1 \%$ & $33.1 \%$ & 0.984 & $41.4 \%$ & $41.3 \%$ & 0.887 & $48.5 \%$ & $48.4 \%$ & 0.900 \\
\hline Diabetes & $6.0 \%$ & $6.1 \%$ & 0.922 & $6.5 \%$ & $6.7 \%$ & 0.940 & $8.5 \%$ & $8.7 \%$ & 0.712 & $11.0 \%$ & $12.0 \%$ & 0.375 \\
\hline Ventilator Dependent & $0.1 \%$ & $0.1 \%$ & 0.577 & $0.1 \%$ & $0.1 \%$ & 0.841 & $0.0 \%$ & $0.1 \%$ & 0.197 & $0.1 \%$ & $0.1 \%$ & 1.000 \\
\hline Hx of severe COPD & $4.5 \%$ & $4.5 \%$ & 0.897 & $4.7 \%$ & $4.7 \%$ & 0.690 & $4.7 \%$ & $4.6 \%$ & 0.426 & $5.0 \%$ & $5.2 \%$ & 0.629 \\
\hline Chronic steroid use & $4.2 \%$ & $4.1 \%$ & 0.460 & $4.1 \%$ & $4.2 \%$ & 0.410 & $3.4 \%$ & $3.4 \%$ & 0.929 & $3.2 \%$ & $3.2 \%$ & 0.887 \\
\hline Dialysis & $1.7 \%$ & $1.6 \%$ & 0.397 & $1.5 \%$ & $1.3 \%$ & 0.030 & $1.2 \%$ & $1.1 \%$ & 0.166 & $1.0 \%$ & $1.0 \%$ & 0.898 \\
\hline \multicolumn{13}{|l|}{ ASA Classification } \\
\hline I-No Disturbance & $17.6 \%$ & $17.5 \%$ & \multirow{4}{*}{0.349} & $12.3 \%$ & $12.3 \%$ & \multirow{4}{*}{0.781} & $5.1 \%$ & $5.2 \%$ & \multirow{4}{*}{0.798} & $2.1 \%$ & $2.1 \%$ & \multirow{4}{*}{0.925} \\
\hline II-Mild Disturbance & $53.1 \%$ & $53.1 \%$ & & $56.2 \%$ & $56.2 \%$ & & $57.2 \%$ & $57.1 \%$ & & $49.7 \%$ & $49.9 \%$ & \\
\hline III-Severe Disturb. & $26.3 \%$ & $26.5 \%$ & & $28.6 \%$ & $28.8 \%$ & & $35.3 \%$ & $35.3 \%$ & & $45.0 \%$ & $44.6 \%$ & \\
\hline IV-Life Threatening & $2.8 \%$ & $2.6 \%$ & & $2.6 \%$ & $2.5 \%$ & & $2.2 \%$ & $2.1 \%$ & & $3.1 \%$ & $3.1 \%$ & \\
\hline
\end{tabular}

Table 5: Multivariate logistic regression for BMI categories associated with 30-day comorbidity outcomes (unmatched samples).

\begin{tabular}{|c|c|c|c|c|c|c|c|c|}
\hline $\begin{array}{l}\text { UNMATCHED } \\
\text { COHORT }\end{array}$ & \multicolumn{2}{|c|}{ Any Morbidity (30-day) } & \multicolumn{2}{|c|}{ Wound Morbidity (30-day) } & \multicolumn{2}{|c|}{$\begin{array}{c}\text { Respiratory Morbidity } \\
\text { (30-day) }\end{array}$} & \multicolumn{2}{|c|}{ Mortality (30-day) } \\
\hline BMI Category & $\begin{array}{c}\text { Odds Ratio } \\
\text { (95\% CI) }\end{array}$ & $P$-value & $\begin{array}{c}\text { Odds Ratio } \\
\text { (95\% CI) }\end{array}$ & $P$-value & $\begin{array}{c}\text { Odds Ratio } \\
(95 \% \text { CI })\end{array}$ & $P$-value & $\begin{array}{c}\text { Odds Ratio } \\
\text { (95\% CI) }\end{array}$ & $P$-value \\
\hline Normal & Reference & & Reference & & \multicolumn{2}{|c|}{ Reference } & \multicolumn{2}{|c|}{ Reference } \\
\hline Pre-obese & $1.08(1.00-1.16)$ & 0.0524 & $1.30(1.17-1.45)$ & $<.0001$ & $0.95(0.82-1.10)$ & 0.51 & $0.61(0.46-0.81)$ & 0.0006 \\
\hline Obese I & $1.33(1.24-1.44)$ & $<.0001$ & $1.82(1.64-2.01)$ & $<.0001$ & $1.00(0.86-1.14)$ & 0.99 & $0.59(0.44-0.80)$ & 0.0005 \\
\hline Obese II & $1.69(1.56-1.83)$ & $<.0001$ & $2.48(2.22-2.77)$ & $<.0001$ & $1.20(1.02-1.41)$ & 0.02 & $0.52(0.36-0.75)$ & 0.0005 \\
\hline Obese III & $2.54(2.35-2.75)$ & $<.0001$ & $3.92(3.52-4.36)$ & $<.0001$ & $1.61(1.38-1.88)$ & $<.0001$ & $0.76(0.53-1.01)$ & 0.124 \\
\hline
\end{tabular}

Table 6: Multivariate logistic regression for BMI categories associated with 30-day comorbidity outcomes matched samples).

\begin{tabular}{|c|c|c|c|c|c|c|c|c|}
\hline $\begin{array}{l}\text { MATCHED COHORT } \\
\text { (5:1 greedy match) }\end{array}$ & \multicolumn{2}{|c|}{ Any Morbidity (30-day) } & \multicolumn{2}{|c|}{$\begin{array}{l}\text { Wound Morbidity (30- } \\
\text { day) }\end{array}$} & \multicolumn{2}{|c|}{$\begin{array}{c}\text { Respiratory Morbidity } \\
\text { (30-day) }\end{array}$} & \multicolumn{2}{|c|}{ Mortality (30-day) } \\
\hline BMI Category & $\begin{array}{l}\text { Odds Ratio } \\
\text { (95\% CI) }\end{array}$ & $P$-value & $\begin{array}{l}\text { Odds Ratio } \\
\text { (95\% CI) }\end{array}$ & $P$-value & $\begin{array}{l}\text { Odds Ratio } \\
\text { (95\% CI) }\end{array}$ & $P$-value & $\begin{array}{l}\text { Odds Ratio } \\
\text { (95\% CI) }\end{array}$ & $P$-value \\
\hline Normal & Reference & & Reference & & \multicolumn{2}{|c|}{ Reference } & \multicolumn{2}{|c|}{ Reference } \\
\hline Pre-obese & $1.12(1.03-1.22)$ & 0.008 & $1.41(1.25-1.58)$ & $<.0001$ & $0.93(0.79-1.01)$ & 0.39 & $0.69(0.49-0.97)$ & 0.03 \\
\hline Obese I & $1.35(1.24-1.47)$ & $<.0001$ & $1.91(1.7-2.15)$ & $<.0001$ & $0.97(0.82-1.16)$ & 0.76 & $0.53(0.36-0.77)$ & 0.001 \\
\hline Obese II & $1.39(1.27-1.52)$ & $<.0001$ & $2.08(1.84-2.36)$ & $<.0001$ & $1.00(0.82-1.19)$ & 0.96 & $0.38(0.24-0.60)$ & $<.0001$ \\
\hline Obese III & $1.64(1.49-1.80)$ & $<.0001$ & $2.75(2.41-3.14)$ & $<.0001$ & $0.98(0.79-1.18)$ & 0.76 & $0.41(0.25-0.64)$ & $<.0001$ \\
\hline
\end{tabular}


acknowledged. Furthermore, the rate of diabetes in our total sample (13.7\%) is above the age-adjusted incidence of diabetes in the United States from 2005-2014 provided by the CDC (7.6\%) [18]. Having an elevated rate of DM present in our sample may have led to confounding prior to propensity score matching. Propensity score-matching analyses accounted for these confounding variables by producing matched-groups of normal BMI vs Pre-/obese I-III BMI patients that no longer had significant differences in their baseline characteristics (Table 4). Distributing these baseline characteristics between the cases (overweight BMI patients) and controls (normal BMI patients) randomly reduced selection bias and allowed for optimal isolation of body mass index as our independent variable. This technique of propensity-score-matching regression analyses has been shown to be one of the closest imitations of a randomized clinical trial $[14,19]$.

Prior to propensity-score matching, multivariate regression showed that the pre-obese BMI group was not associated with an increased likelihood of suffering any post-operative complication (Table 5). However, patients with obese I to obese III BMIs were found to have a positive association with developing any complication in the 30 -day post-operative period. Looking specifically at wound complications such as SSI, every BMI category above the normal BMI level was associated with an increased likelihood of suffering a 30day wound morbidity. However, in regards to respiratory morbidities, only patients in the obese III $\left(\geq 40 \mathrm{~kg} / \mathrm{m}^{2}\right)$ were found to be positively associated. An interesting association was found between pre-obese, obese I, obese II patients and death within 30-days of VHR. These three groups were found to have a reduced likelihood of dying compared to patients in the normal BMI group. Given the confounding of covariates in this analyses, some of the associations between increasing BMI category and adverse 30-day outcomes may simply be due to these comorbidities instead.

As mentioned earlier, some of the associations between BMI categories and 30-day outcomes in the unmatched analyses may simply be due to the confounding comorbidities present in the sample. However, propensity-score matching reduced this selection bias and allowed for optimal isolation of $\mathrm{BMI}$ as a predictor of adverse 30-day outcomes via multivariate logistic regression. Every BMI category was now significantly associated with an increased likelihood of developing any morbidity in the 30-day post-operative period (Table 6). More importantly, there is a "dose-dependent" relationship between increasing BMI and the odds of suffering any 30day complication. This is relevant clinically as patients in a lower BMI category can expect their odds of developing any surgical complication post-VHR to increase as they advance to higher and more morbidly obese BMI categories. These findings draw only some parallels with the findings of a recent study by Pernar et al that looked at only a subset of the ventral hernia CPT codes that were used in our current study. Pernar et al. found that only patients above $40 \mathrm{~kg} / \mathrm{m}^{2}$ have an increased likelihood of developing any morbidity after open ventral hernia repair $\left(\mathrm{OR}_{\text {OBESE-III }} 3.40, p=0.004\right)$ [8]. This is consistent with our finding that patients in the obese III group $\left(>40 \mathrm{~kg} / \mathrm{m}^{2}\right)$ also have increased odds of developing any 30 -day morbidity $\left(\mathrm{OR}_{\text {OBESE-III }} 1.64, p<.0001\right)$. The lack of propensityscore matching and the exclusion of laparoscopic ventral hernia CPT codes contribute to not only the inflated odds ratio in the Pernar et al. study, but also explain why they did not find a significant association between other obese groups.

Even though we were still able to see a dose-dependent association between every BMI category and the likelihood of suffering a wound complication (Table 6), the ORs that were produced in the matched analyses were not as high as the unmatched analyses. This can be explained by the fact that greater frequencies of diabetes and pre-operative cardiac risk in the pre-obese/obese I-III groups in the unmatched analyses were contributing to the presence of increased wound morbidity and were thus, inflating the ORs associated with greater obesity groups. Removing the bias of these confounding variables by distributing them evenly amongst normal BMI and pre-obese/obese I-III groups shows that BMI is an independent risk factor for 30-day wound complications following VHR. This is consistent with recent literature that found associations between obesity and 30-day wound-related complications $[8,12]$. Propensity-score matching removed the association between obese III patients and their likelihood of a 30-day respiratory morbidity (pneumonia, unplanned intubation, $\mathrm{PE}$, ventilator dependence $\geq 48 \mathrm{~h}$ ). Similar to the findings with 30-day wound-morbidity, the association that was seen in unmatched analyses can be attributed to the confounding pre-operative variables (i.e., history of COPD, ventilator dependence) that were contributing to a respiratory morbidity. Lastly, even after propensity-matching, patients in all overweight BMI categories still had a reduced likelihood of 30-day mortality compared to patients in the normal BMI category, giving merit to a popular theory in obesity-outcome literature called the "obesity paradox". The relationship between increasing BMI and reduced likelihood of death after surgery has been reproduced in previous hernia literature as well $[10,20]$

Obesity is widely accepted to have adverse effects on diabetes, cardiovascular disease, orthopedic procedures, thoracic surgery, vascular surgery, cancer, and life expectancy $[9,10]$. However, over the past 15 years, there has been growing evidence indicating that patients with elevated BMI may actually have diminished rates of mortality. Initially discovered by Gruberg et al. 15 years ago as a phenomenon found only to coronary heart disease [21], the obesity paradox has been spread to non-cardiovascular disciplines as well $[22,23]$. Whereas some systematic reviews have shown the paradox to only apply to patients with pre-obese BMI (BMI $25-30 \mathrm{~kg} / \mathrm{m}^{2}$ ) [24], other studies have found diminished rates of mortality for all obesity categories. A landmark study by Mullen et al. on mortality in patients after non-bariatric general surgery (including VHR) found a lower risk of 30day mortality in pre-obese and obese I-II patients compared to those with normal BMI [23]. Our findings reproduce the obesity paradox across all Obesity categories and show a significant reduced likelihood of 30-day mortality after VHR compared to normal BMI patients. However, the extremely low frequency of overall 30-day mortality in our total cohort (<1\%) overall combined with the fact that there were simply 
a greater number of deaths in normal BMI patients compared to the obese categories indicate that the obesity paradox we found for VHR may lack statistical power.

\section{Study Limitations}

The analyses presented has a number of limitations related to the use of the National Surgical Quality Improvement Program (NSQIP). The retrospective nature of our study combined with the fact that NSQIP collects data prospectively impairs our ability to make an unequivocal cause and effect relationship between obesity and adverse 30-day surgical outcomes. In addition, by collecting outcomes only in the 30-day post-operative window, NSQIP fails to capture any adverse surgical outcomes that may present later. Furthermore, NSQIP fails to collect information on patients who presented with ventral hernia but did not undergo surgical treatment. Lastly, NSQIP lacks specific details related to obesity, such as measurement metrics and diabetes information. Body mass index, while widely used due to ease of measurement and standardization, is an indirect and imperfect measurement of obesity compared to metrics that were not found in NSQIP such as body fat percentage and waist-to-hip circumference. BMI does not distinguish between body fat and lean body mass and is furthermore, limited by the inherent differences in body fat between men and women as well as between younger patients and the elderly. Diabetes information in NSQIP is also restricted to only therapeutic metrics and does not provide information on the type of diabetes or the onset of diabetes in the patient. This information could help us further refine our findings by allowing for a discussion on the impact of diabetes related to obesity on adverse surgical outcomes. Furthermore, NSQIP lacked information on certain surgery-specific data such as hernia gap size that have been shown to be associated positively with post-operative complications [25].

In addition to the shortcomings of the NSQIP database, there are a number of statistical limitations that are worth mentioning as well. Even though propensity score matching reduces the likelihood of selection bias, it is not a complete randomized clinical study. This means that even though we included all the preoperative variables we thought to be confounding in our propensity-score match, there could still be other factors that a patient has that are contributing to adverse outcomes that is lacking in NSQIP.

\section{Conclusion}

This study analyzed over 160,00 patients undergoing elective ventral hernia repair from a national, validated surgical database. We used propensity score matching to quantify the isolated impact of increasing BMI on the likelihood of a patient undergoing ventral hernia repair to develop an adverse surgical outcome in the 30-day post-operative window. Even though patients with normal BMI had the lowest risk of a 30-day complication, the majority of patients (> 80\%) in our study undergoing VHR were overweight or obese. This finding, in combination with projections that estimate that $50-85 \%$ of adults will be overweight or obese in the United States by 2030 [2, 26] suggest that surgeons will be increasingly called upon to manage VHR in the future. We found that having a BMI greater than 25 increases the likelihood of developing any surgical complication after VHR (includes wound-, respiratory-, cardiac-, genitourinary, CNSrelated complications). Furthermore, we found this association to be dose-dependent, as patients in increasingly obese BMI categories were found to have a higher likelihood of suffering any adverse post-operative complications. Looking specifically at wound and respiratory complications, we found that the association between 30-day respiratory complications and the morbidly obese to be confounded by other comorbidities found in the morbidly obese population. Propensity score matching removed this association and more importantly, allowed us to discover that $\mathrm{BMI}$ is an independent risk factor for developing adverse 30-day wound complications in a dose-dependent manner. The results of this study implicate rising BMI with higher odds of overall and wound morbidity and validate the rise in preoperative optimization such as targeted weight loss that has been occurring in elective surgical procedures such as VHR. It is therefore beneficial for surgeons to be aware of the isolated impact BMI can have on post-operative complications after VHR and use this information to better counsel their patients about the risks of elective ventral hernia surgery.

Our study included all modes of ventral hernia repair, including open (OVHR) and laparoscopic (LVHR) methods used from 2005-2014. A recent NSQIP study by Regner et al found that Laparoscopic VHR was better than Open VHR at reducing wound complications in obese patients from 2009-2012 [9]. It would be appropriate to expand upon this study and include procedures from additional years (20052014) as well as investigate the potential benefits of LVHR in reducing other surgical outcomes such as cardiac or respiratory morbidities as well.

\section{Conflict of Interest}

$\mathrm{NB}$ and $\mathrm{AM}$ declare no conflict of interest.

\section{References}

1. Nguyen MT, Berger RL, Hicks SC, Davila JA, Li LT, et al. 2014. Comparison of outcomes synthetic mesh vs suture repair of elective primary ventral herniorrhaphy: a systematic review and metaanalysis. JAMA Surg 149(5): 415-421. doi: https://dx.doi.org/10.1001/ jamasurg.2013.5014

2. Hruby A, Hu FB. 2015. The epidemiology of obesity: a big picture. Pharmacoeconomics 33(7): 673-689. doi: https://dx.doi.org/10.1007/ s40273-014-0243-x

3. Ooms LS, Verhelst J, Jeekel J, Ijzermans JN, Lange JF, et al. 2016. Incidence, risk factors, and treatment of incisional hernia after kidney transplantation: an analysis of 1,564 consecutive patients. Surgery 159(5): 1407-1411. doi: https://dx.doi.org/10.1016/j.surg.2015.11.017

4. Sorensen LT, Hemmingsen UB, Kirkeby LT, Kallehave F, Jorgensen LN. 2005. Smoking is a risk factor for incisional hernia. Arch Surg 140(2): 119-123. doi: https://dx.doi.org/10.1001/archsurg.140.2.119

5. Sugerman HJ, Kellum JM Jr., Reines HD, DeMaria EJ, Newsome $\mathrm{HH}$, et al. 1996. Greater risk of incisional hernia with morbidly obese than steroid-dependent patients and low recurrence with prefascial polypropylene mesh. Am J Surg 171(1): 80-84. doi: https://dx.doi. org/10.1016/S0002-9610(99)80078-6 
6. Sauerland S, Korenkov M, Kleinen T, Arndt M, Paul A. 2004. Obesity is a risk factor for recurrence after incisional hernia repair. Hernia 8(1): 42-46. doi: https://dx.doi.org/10.1007/s10029-003-0161-x

7. Poulose BK, Shelton J, Phillips S, Moore D, Nealon W, et al. 2012. Epidemiology and cost of ventral hernia repair: making the case for hernia research. Hernia 16(2): 179-183. doi: https://dx.doi.org/10.1007/ s10029-011-0879-9

8. Pernar LI, Pernar CH, Dieffenbach BV, Brooks DC, Smink DS, et al. 2016. What is the BMI threshold for open ventral hernia repair? Surg Endosc 31(3): 1311-1317. doi: https://dx.doi.org/10.1007/s00464-016-5113-5

9. Regner JL,Mrdutt MM,Munoz-Maldonado Y.2015.Tailoring surgical approach for elective ventral hernia repair based on obesity and National Surgical Quality Improvement Program outcomes. Am J Surg 210(6): 1024-1029. doi: https://dx.doi.org/10.1016/j.amjsurg.2015.08.001

10. Palvannan P, Miranda I, Merchant AM. 2016. The combined effect of age and body mass index on outcomes in foregut surgery: a regression model analysis of the National Surgical Quality Improvement Program data. Surg Endosc 30(6): 2572-2582. doi: https://dx.doi.org/10.1007/ s00464-015-4529-7

11. Raftopoulos I, Courcoulas AP. 2007. Outcome of laparoscopic ventral hernia repair in morbidly obese patients with a body mass index exceeding $35 \mathrm{~kg} / \mathrm{m}^{2}$. Surg Endosc 21(12): 2293-2297. doi: https://dx.doi. org/10.1007/s00464-007-9406-6

12. Cox TC, Blair LJ, Huntington CR, Colavita PD, Prasad T, et al. 2016. The cost of preventable comorbidities on wound complications in open ventral hernia repair. J Surg Res 206(1): 214-222. doi: https://dx.doi. org/10.1016/j.jss.2016.08.009

13. Ingraham AM, Richards KE, Hall BL, Ko CY. 2010. Quality improvement in surgery: the American College of Surgeons National Surgical Quality Improvement Program approach. Adv Surg 44: 251-267.

14. Austin PC. 2011. An introduction to propensity score methods for reducing the effects of confounding in observational studies. Multivariate Behav Res 46(3): 399-424. doi: https://dx.doi.org/10.1080 /00273171.2011.568786

15. Parsons LS. 2004. Performing a $1: \mathrm{N}$ case-control match on propensity score. In: Proceedings of the $29^{\text {th }}$ annual SAS users group international conference, Canada.

16. Anaya DA, Dellinger EP. 2006. The obese surgical patient: a susceptible host for infection. Surg Infect (Larchmt) 7(5): 473-480. doi: https:// dx.doi.org/10.1089/sur.2006.7.473
17. Kabon B, Nagele A, Reddy D, Eagon C, Fleshman JW, et al. 2004. Obesity decreases perioperative tissue oxygenation. Anesthesiology 100(2): 274-280

18. Shrestha SS, Thompson TJ, Kirtland KA, Gregg EW, Beckles GL, et al. 2016. Changes in disparity in county-level diagnosed diabetes prevalence and incidence in the united states, between 2004 and 2012. PLoS One 11(8): e0159876. doi: https://dx.doi.org/10.1371/journal. pone. 0159876

19. Seicean A, Alan N, Seicean S, Worwag M, Neuhauser D, et al. 2014. Impact of increased body mass index on outcomes of elective spinal surgery. Spine (Phila Pa 1976) 39(18): 1520-1530. doi: https://dx.doi. org/10.1097/BRS.0000000000000435

20. Chung PJ, Lee JS, Tam S, Schwartzman A, Bernstein MO, et al. 2016. Predicting 30-day postoperative mortality for emergent anterior abdominal wall hernia repairs using the American College of Surgeons National Surgical Quality Improvement Program database. Hernia. doi: https://dx.doi.org/10.1007/s10029-016-1538-y

21. Gruberg L, Weissman NJ, Waksman R, Fuchs S, Deible R, et al. 2002. The impact of obesity on the short-term and long-term outcomes after percutaneous coronary intervention: the obesity paradox? J Am Coll Cardiol 39(4): 578-584.

22. Hainer V, Aldhoon-Hainerova I. 2013. Obesity paradox does exist. Diabetes Care 36(Suppl 2): S276-281. doi: https://dx.doi.org/10.2337/ dcS13-2023

23. Mullen JT, Moorman DW, Davenport DL. 2009. The obesity paradox: body mass index and outcomes in patients undergoing nonbariatric general surgery. Ann Surg 250(1): 166-172. doi: https://dx.doi. org/10.1097/SLA.0b013e3181ad8935

24. Romero-Corral A, Montori VM, Somers VK, Korinek J, Thomas RJ, et al. 2006. Association of bodyweight with total mortality and with cardiovascular events in coronary artery disease: a systematic review of cohort studies. Lancet 368(9536): 666-678. doi: https://dx.doi. org/10.1016/S0140-6736(06)69251-9

25. Dietz UA, Winkler MS, Hartel RW, Fleischhacker A, Wiegering A, et al. 2014. Importance of recurrence rating, morphology, hernial gap size, and risk factors in ventral and incisional hernia classification. Hernia 18(1): 19-30. doi: https://dx.doi.org/10.1007/s10029-012-0999-x

26. Finkelstein EA, Khavjou OA, Thompson H, Trogdon JG, Pan L, et al. 2012. Obesity and severe obesity forecasts through 2030. Am J Prev Med 42(6): 563-570. doi: https://dx.doi.org/10.1016/j.amepre.2011.10.026 